\title{
Solar energetic particle heavy ion properties in the widespread event of 2020 November 29
}

G. M. Mason ${ }^{1} \oplus$, C. M. S. Cohen ${ }^{2}$, G. C. Ho ${ }^{1}$, D. G. Mitchell ${ }^{1}$, R. C. Allen ${ }^{1}$, M. E. Hill ${ }^{1}$, G. B. Andrews ${ }^{1}$, L. Berger ${ }^{3}$, S. Boden ${ }^{3}$, S. Böttcher ${ }^{3}$, I. Cernuda ${ }^{4}$, E. R. Christian ${ }^{5}$, A. C. Cummings ${ }^{2}$, A. J. Davis ${ }^{2}$, M. I. Desai ${ }^{6}$, G. A. de Nolfo ${ }^{5}$, S. Eldrum ${ }^{3}$, R. Elftmann ${ }^{3}$, A. Kollhoff ${ }^{3}$, J. Giacalone ${ }^{7}$, R. Gómez-Herrero ${ }^{4}$, J. Hayes ${ }^{1}$, N. P. Janitzek ${ }^{8}$, C. J. Joyce ${ }^{9}$, A. Korth ${ }^{10}$, P. Kühl ${ }^{3}$, S. R. Kulkarni ${ }^{3}$, A. W. Labrador ${ }^{2}$, F. Espinosa Lara ${ }^{4}$, W. J. Lees ${ }^{1}$, R. A. Leske ${ }^{2}$, U. Mall ${ }^{10}$, C. Martin ${ }^{3}$, A. Martínez Hellín ${ }^{3}$, W. H. Matthaeus ${ }^{11}$, D. J. McComas ${ }^{9}$, R. L. McNutt Jr. ${ }^{1}$, R. A. Mewaldt ${ }^{2}$, J. G. Mitchell ${ }^{5,12}$, D. Pacheco ${ }^{3}$, P. Parra Espada ${ }^{3}$, M. Prieto ${ }^{4}$, J. S. Rankin ${ }^{9}$, A. Ravanbakhsh ${ }^{3,10}$, J. Rodríguez-Pacheco ${ }^{4}$, O. Rodríguez Polo $^{4}$, E. C. Roelof ${ }^{1}$, S. Sánchez-Prieto ${ }^{4}$, C. E. Schlemm ${ }^{1}$, N. A. Schwadron ${ }^{13}$, H. Seifert ${ }^{1}$, E. C. Stone ${ }^{2}$, J. R. Szalay ${ }^{9}$, J. C. Terasa ${ }^{3}$, K. Tyagi ${ }^{1}$, J. L. Freiherr von Forstner ${ }^{3}$, M. E. Wiedenbeck ${ }^{14}$, R. F. Wimmer-Schweingruber ${ }^{3}$, Z. G. $\mathrm{Xu}^{3}$, and M. Yedla ${ }^{3,10}$

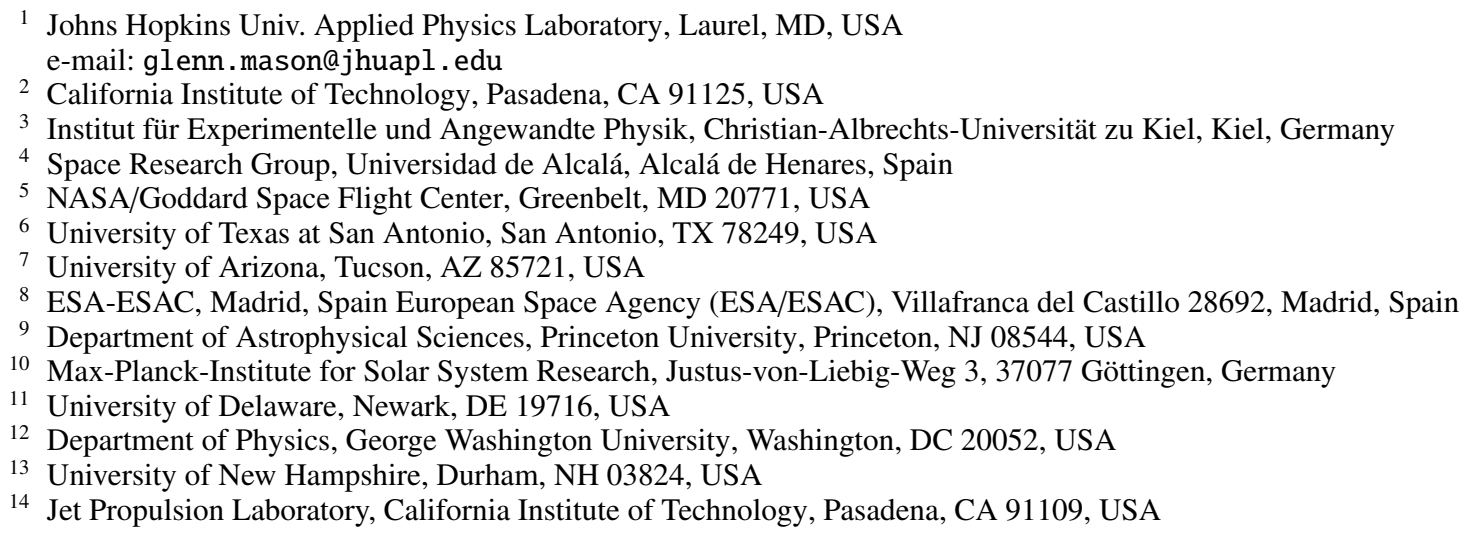

Received 13 May 2021 / Accepted 17 June 2021

\section{ABSTRACT}

\begin{abstract}
Context. Following a multi-year minimum of solar activity, a solar energetic particle event on 2020 Nov. 29 was observed by multiple spacecraft covering a wide range of solar longitudes including ACE, the Solar Terrestrial Relations Observatory-A, and the recently launched Parker Solar Probe and Solar Orbiter.

Aims. Multi-point observations of a solar particle event, combined with remote-sensing imaging of flaring, shocks, and coronal mass ejections allows for a global picture of the event to be synthesized, and made available to the modeling community to test, constrain, and refine models of particle acceleration and transport according to such parameters as shock geometries and particle mass-to-charge ratios.

Methods. Detailed measurements of heavy ion intensities, time dependence, fluences, and spectral slopes provided the required test data for this study.

Results. The heavy ion abundances, timing, and spectral forms for this event fall well within the range found in prior surveys at 1 au. The spectra were well fitted by broken power law shapes; the $\mathrm{Fe} / \mathrm{O}$ ratio was somewhat lower than the average of other events. In addition, ${ }^{3} \mathrm{He} /{ }^{4} \mathrm{He}$ was very low, with only the upper limits established here.
\end{abstract}

Key words. acceleration of particles - Sun: abundances - Sun: flares - Sun: particle emission

\section{Introduction}

A major focus of solar energetic particle (SEP) research addresses major events that are capable of filling most or nearly all of the inner solar system with ionizing radiation. Recently, the Solar Orbiter mission (Müller et al. 2020), launched February 2020 , added a new vantage point to the fleet of spacecraft studying widespread SEP events. On 29 Nov. 2020, an M4.4 solar X-ray flare and the accompanying coronal mass ejection (CME) and shock provided the first opportunity for a global study using Solar Orbiter.

Since this event occurred after a several-year hiatus in solar activity, initial studies have already appeared, in particular, the work of Kollhoff et al. (2021) describing the solar activity with 
Table 1. 29 Nov. 2020 event.

\begin{tabular}{|c|c|c|c|c|c|c|c|c|c|c|}
\hline \multirow[t]{2}{*}{ Spacecraft } & \multirow{2}{*}{$\begin{array}{l}\text { s/c Carr } \\
\text { long. } \\
\left(^{\circ}\right)\end{array}$} & \multirow{2}{*}{$\begin{array}{c}\text { s/c radial } \\
\text { dist } \\
(\mathrm{au})\end{array}$} & \multirow{2}{*}{$\begin{array}{l}\text { s/c foot- } \\
\text { point } \\
(1) \\
\left({ }^{\circ}\right) \\
\end{array}$} & \multirow{2}{*}{$\begin{array}{c}\Delta \text { Long. flare-s/c } \\
\text { footpoint }{ }^{(2)} \\
\left({ }^{\circ}\right) \\
\end{array}$} & \multicolumn{3}{|c|}{ Fluence ${ }^{(3)}$} & \multirow{2}{*}{$\begin{array}{c}\text { Fluence } \\
\text { period }^{(4)}\end{array}$} & \multirow{2}{*}{$\begin{array}{l}\text { Shock } \\
\text { time }\end{array}$} & \multirow[t]{2}{*}{ Instrument } \\
\hline & & & & & $\mathrm{He}$ & $\mathrm{O}$ & $\mathrm{Fe}$ & & & \\
\hline Solar Orbiter & 110 & 0.88 & 160 & 89 & $\ldots$ & & & $335.0-337.5$ & & $\mathrm{EPD} / \mathrm{SIS}^{(5)}$ \\
\hline PSP & 252 & 0.81 & 298 & -48 & 3900 & 57 & 11 & $334.5-339.708$ & $18: 35$ Nov. $30^{(6)}$ & ISOIS/EPI-Lo EPI-Hi ${ }^{(7)}$ \\
\hline STEREO-A & 290 & 0.96 & 345 & -96 & 7083 & 123 & 36 & $335.5-339.5$ & 07:25 Dec. $1^{(2)}$ & IMPACT/SIT, LET ${ }^{(8)}$ \\
\hline $\mathrm{ACE}$ & 348 & 0.98 & 404 & -155 & 1466 & 7.0 & 1.2 & $335.0-342.5$ & $\ldots$ & ACE/ULEIS, SIS ${ }^{(9)}$ \\
\hline
\end{tabular}

Notes. ${ }^{(1)} \mathrm{In} \mathrm{CL}$ assuming $400 \mathrm{~km} \mathrm{~s}^{-1}$ solar wind speed, (2) flare at CL 249 $\quad$ (Kollhoff et al. 2021), ${ }^{(3)} \sim 0.27 \mathrm{MeV}$ nucleon $^{-1} \times 10^{4}$ $\left(\mathrm{cm}^{-2}\right.$ sr MeV nucleon $\left.{ }^{-1}\right),{ }^{(4)}$ day of 2020, ${ }^{(5)}$ Rodríguez-Pacheco et al. $(2020),{ }^{(6)}$ Cohen et al. (2021), ${ }^{(7)}$ McComas et al. (2014), ${ }^{(8)}$ Luhmann et al. (2008), Mason et al. (2008), Mewaldt et al. (2008), ${ }^{(9)}$ Stone et al. (1998a,b), Mason et al. (1998).

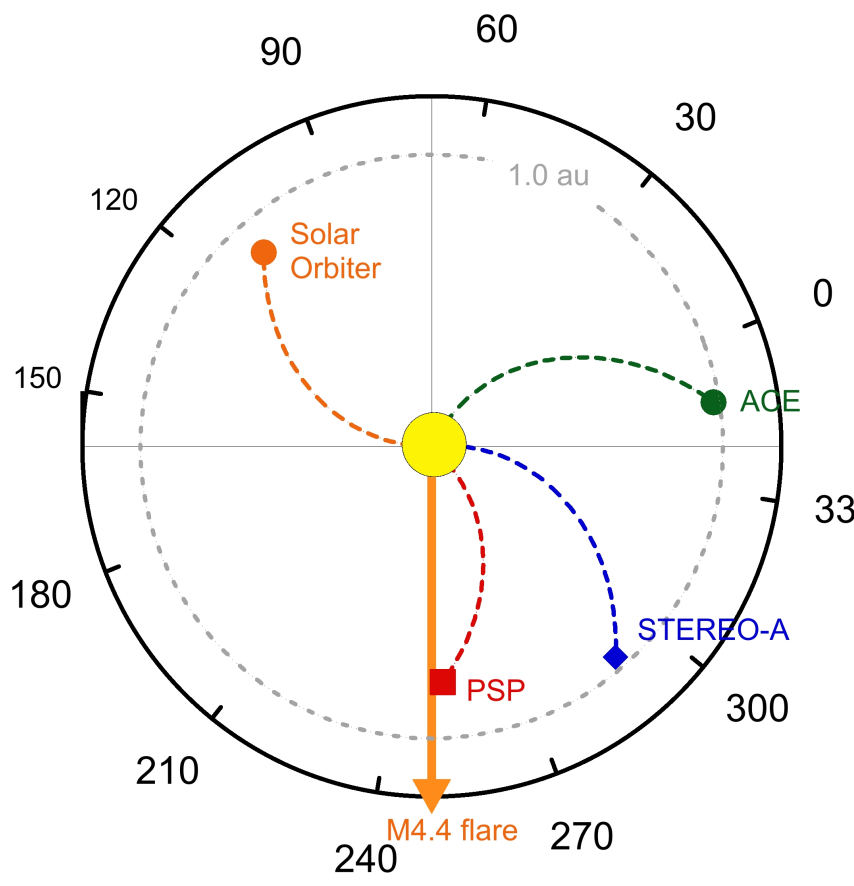

Fig. 1. Spacecraft locations in Carrington longitude and heliocentric radius at the time of 29 Nov. 2020 flare. Magnetic spirals assume $400 \mathrm{~km} \mathrm{~s}^{-1}$ solar wind speed.

an emphasis on shock properties, as well as proton and electron data to determine particle injection timing at or near the Sun. Cohen et al. (2021) have described the energetic particle observations from Parker Solar Probe (PSP), including spectral forms for $\mathrm{H}, \mathrm{He}, \mathrm{O}$, and $\mathrm{Fe}$. In this Letter we examine major heavy ion composition observations from multiple sites (Solar Orbiter, PSP, STEREO-A, and ACE) in order to contribute additional information on the acceleration and transport processes in this event and show its relationship to SEP events surveyed in the prior two solar cycles.

\section{Observations}

Figure 1 shows spacecraft locations at the onset time of the M4.4 flare, at Carrington longitude $249^{\circ}$, beginning 12:34 UT on 29 Nov. 2020. In Table 1, Cols. 1-3 list the spacecraft positions and Col. 4 lists the spacecraft magnetic footpoints assuming a $400 \mathrm{~km} \mathrm{~s}^{-1}$ solar wind speed. Column 5 shows the longitudinal separation of the flare location minus the spacecraft magnetic field footpoints (negative $=$ the flare is eastward of the spacecraft footpoint). Columns 6-8 list the event total fluences for $\mathrm{He}$,

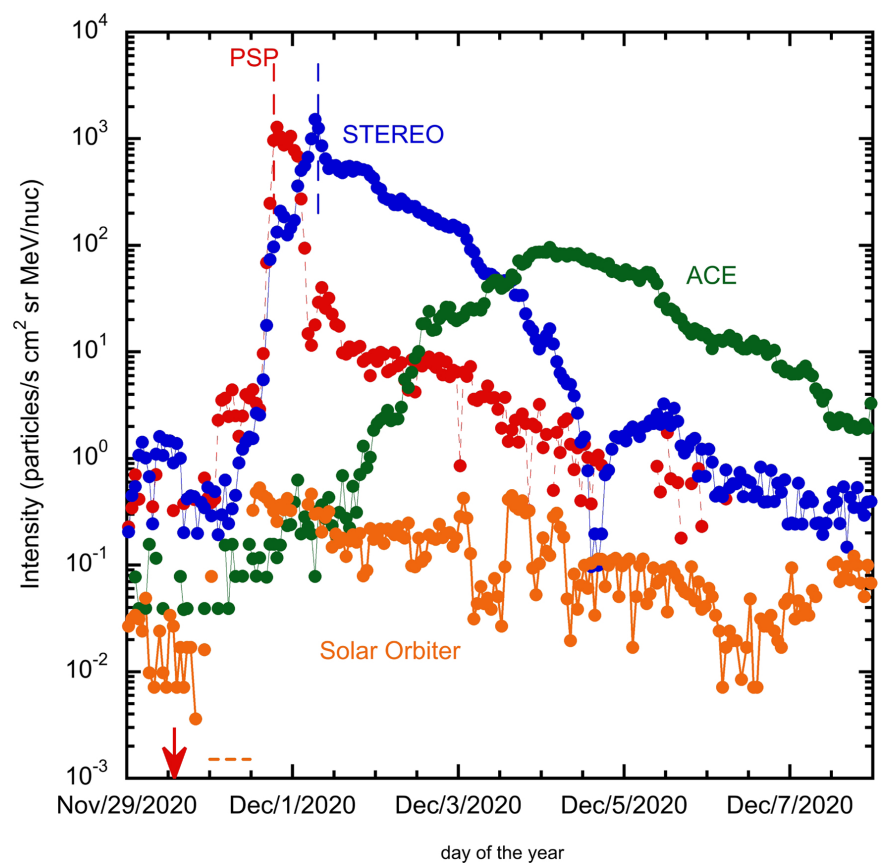

Fig. 2. Hourly average $273 \mathrm{keV}$ nucleon ${ }^{-1} \mathrm{He}$ intensities at each spacecraft. Red downward arrow shows flare time; dashed lines through STEREO and PSP intensities show approximate times of shock passages. Horizontal dashed orange line indicates the data gap in the Solar Orbiter data.

$\mathrm{O}$, and Fe over the energy interval $0.23-0.32 \mathrm{MeV}$ nucleon $^{-1}$. Column 9 lists the time periods for summing fluences. Column 10 lists tentative shock passage times, and Col. 11 lists the instruments used for the energetic particle measurements. Since this event produced a CME and shocks, particle acceleration likely occurred over a range of locations and times as accelerating shocks moved away from the flare site (Cohen et al. 2021; Kollhoff et al. 2021). Event total fluences are not available from the Solar Orbiter instrument since it missed the peak of the intensity due to a software maintenance interval from 0:02 to 11:45 on 30 November. Also, multi-MeV nucleon ${ }^{-1} \mathrm{H}$ and $\mathrm{He}$ data from the PSP instrument are not available for the full event period due to instrumental effects, as discussed in Cohen et al. (2021).

\subsection{Hourly average intensities}

Figure 2 shows hourly intensities at each spacecraft (s/c). The particle rise times show typical forms for low energy ions with the flare located at different longitudes relative to each s/c-Sun line, with slower onsets where the longitude separation is greater. 

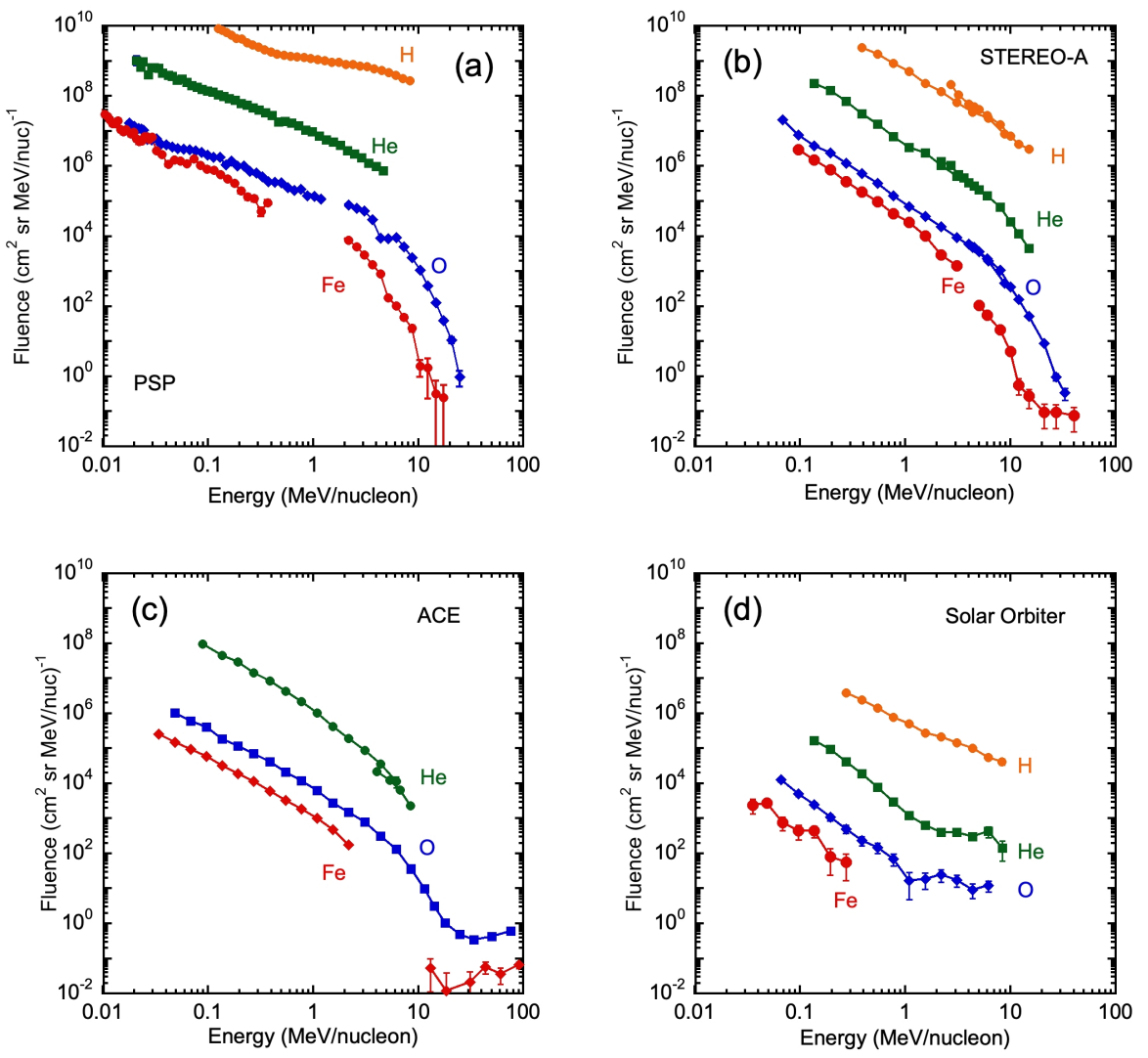

Fig. 3. Fluence spectra for $(a)$ PSP, $(b)$ STEREO-A, (c) ACE, and (d) Solar Orbiter in the 29 Nov. 2020 event.

The Solar Orbiter instrument data gap is marked by the horizontal orange dashed line in the figure. Shock passage times are from Kollhoff et al. (2021) and Cohen et al. (2021).

\subsection{Spectra for $\mathrm{H}, \mathrm{He}, \mathrm{O}$, and $\mathrm{Fe}$}

The fluence spectra summed over the intensity increases at each spacecraft are shown in Fig. 3. The PSP spectra in panel a show clear spectral breaks for $\mathrm{O}$ and $\mathrm{Fe}$, but due to the missing higherenergy $\mathrm{H}$ and $\mathrm{He}$, no breaks are observed for those spectra. The Solar Terrestrial Relations Observatory-A (STEREO-A) spectra in panel $\mathrm{b}$ show clear spectral breaks for $\mathrm{He}, \mathrm{O}$, and $\mathrm{Fe}$, with $\mathrm{Fe}$ ions having a break at lower energy per nucleon than the lighter ions. The ACE spectra in panel c show a clear break for $\mathrm{O}$, and are consistent with a break for $\mathrm{Fe}$ in the gap between $\sim 3$ and $10 \mathrm{MeV} \mathrm{nuc}^{-1}$. The He spectrum also suggests a break, but does not go high enough in energy to determine it accurately. The Solar Orbiter spectra in d flatten above $\sim 1 \mathrm{MeV}$ nucleon $^{-1}$ due to the data gap that occurred during the early rise phase of the event. At lower energies, they are dominated by the decay phase, with the overall fluence lowered because of the gap. Spectral forms and breaks are further discussed in the appendix.

\subsection{Fe/O ratio vs. energy}

Figure 4 shows $\mathrm{Fe} / \mathrm{O}$ ratios as a function of energy for each spacecraft. The PSP ratio decreases almost monotonically with increasing energy. The ACE and STEREO ratios are nearly constant below $1 \mathrm{MeV}$ nucleon $^{-1}$, with STEREO Fe/O significantly higher than at ACE and Solar Orbiter. Above a few $\mathrm{MeV}_{\text {nucleon }^{-1}}$, the ratios decrease rapidly due to the Fe spectrum turning over at lower energy per nucleon than $\mathrm{O}$.

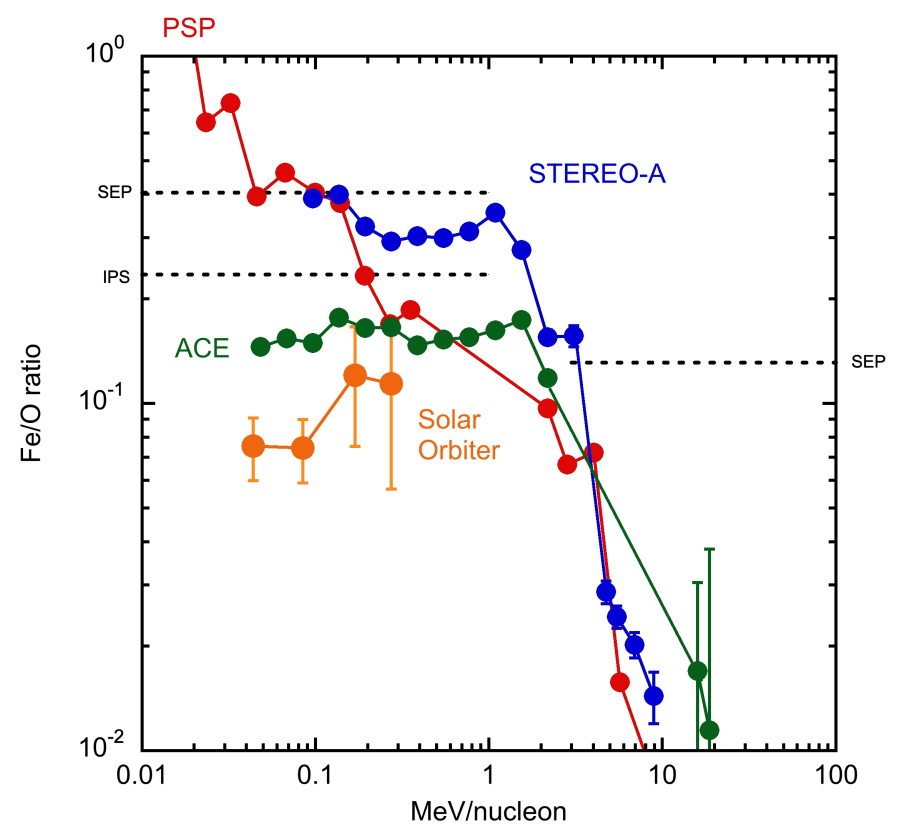

Fig. 4. Fe/O ratios as a function of energy nucleon ${ }^{-1}$. Black dotted lines are large SEP (Desai et al. 2006) and Interplanetary Shock (Desai et al. 2003) survey averages below $1 \mathrm{MeV}$ nucleon $^{-1}$ and SEP averages at a few MeV nucleon ${ }^{-1}$ (Reames 2020).

\subsection{Average abundances}

The average abundances relative to $\mathrm{O}$ over the range $0.32-0.45 \mathrm{MeV}$ nucleon ${ }^{-1}$ are plotted in Fig. 5 with respect to the survey of 64 large SEP events by Desai et al. (2006). PSP 


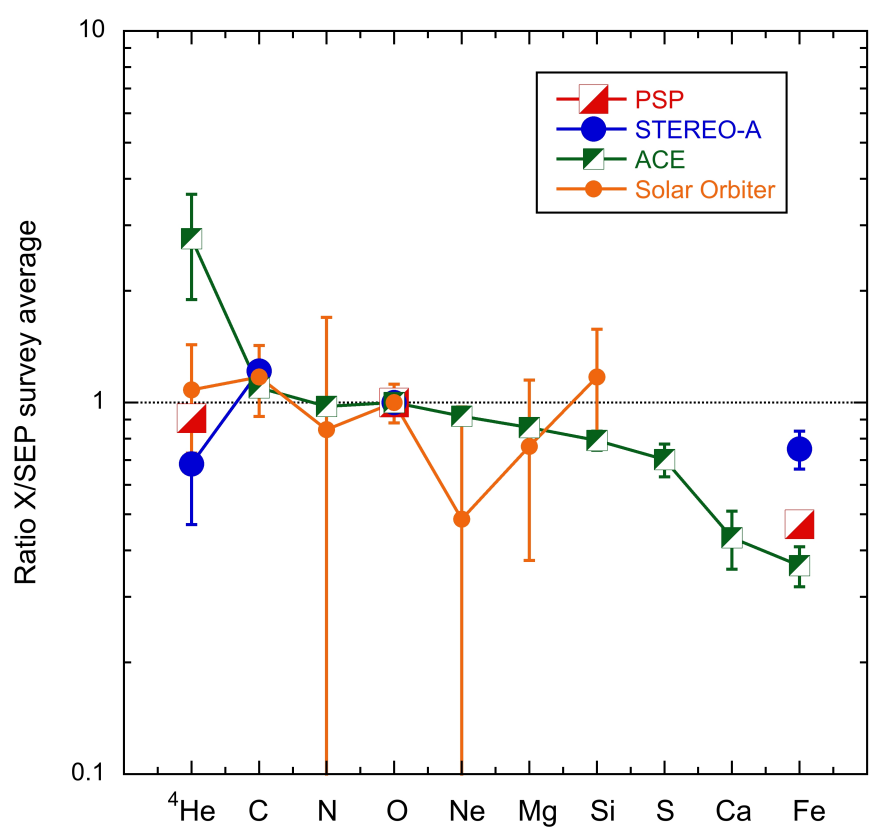

Fig. 5. Abundances $\left(\mathrm{O}=1\right.$.) averaged over $0.32-0.45 \mathrm{MeV}$ nucleon $^{-1}$ for comparison with the same energy range in the survey average of Desai et al. (2006).

and ACE show Fe/O $\sim 2 \times$ lower than average; STEREO-A is slightly below average. The Solar Orbiter heavy ion values up to $\mathrm{Si}$ are consistent with the survey average, although they have large statistical errors.

\section{Discussion and conclusions}

The 29 Nov. 2020 event has properties similar to large CMEassociated SEP events surveyed in previous solar cycles. Most fundamentally, the intensity rise times are reasonably fast for PSP and STEREO, whereas for the more longitudinally distant ACE spacecraft, the rise was slow and intensities did not peak until about three days after the PSP and STEREO peaks. The sharp rise at PSP was followed by a sharp drop several hours later, corresponding to the shock passage and the start of a magnetic cloud passage, respectively (see Cohen et al. 2021). Solar Orbiter, although it is located farther from the flare site in degrees longitude than ACE, saw this event as a western hemisphere flare, with a fast rise for multi-MeV protons (Kollhoff et al. 2021) as well as heavy ions whose high-energy portion was seen by the Solar Orbiter instrument (before its data gap). These general properties of rise time and intensities have been established in large SEP surveys by numerous works (Cane et al. 1988; Cane \& Lario 2006; Cohen et al. 2017; Richardson et al. 2014).

Figure 6 compares the event averaged fluences for the Nov. 29 event with the 41 events surveyed by Cohen et al. (2017). Thick lines in the figure are periodic Gaussian fits to events observed by two of the three spacecraft (see Cohen et al. 2017, for details). The PSP and STEREO-A fluences reported here are close to the mean fit values from the survey, while the ACE fluences for $\mathrm{O}$ and $\mathrm{Fe}$ are lower than the fit means by a factor of 5-10, thus showing a narrower longitudinal distribution than the mean fits for events observed by two spacecraft. Ten events in the survey from Cohen et al. (2021) were observed by three spacecraft and these had a narrower Gaussian width than the 2-event spacecraft events (e.g., $44 \pm 13^{\circ}$ for 3 -spacecraft events

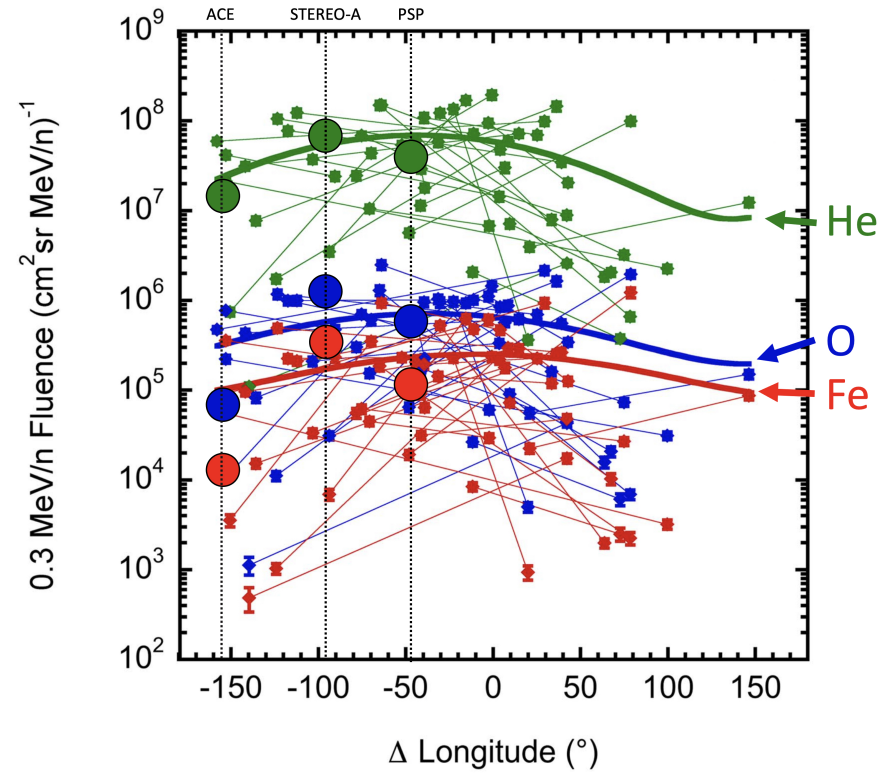

Fig. 6. Plot of $0.3 \mathrm{MeV}$ nucleon $^{-1}$ SEP event fluences vs. flarespacecraft footpoint longitude separation from the 41-event survey of Cohen et al. (2017). Thin lines connect observations at two spacecraft for a single event (filled diamonds). Thick lines are Gaussian fits to each distribution (see text for details). Green: He, Blue: Oxygen, Red: Fe. Large filled circles: He, O, and Fe fluences for the 29 Nov. 2020 event.

vs. $97 \pm 28^{\circ}$ for 2 -spacecraft events for $0.3 \mathrm{MeV}$ nucleon ${ }^{-1} \mathrm{Fe}$ ). Overall, the ACE fluences lie within the range of longitudinal variation seen in the Cohen et al. survey. We note that the Solar Orbiter data are not shown since the data gap precludes fluence measurements.

The PSP and ACE heavy ion abundances (normalized to O) in Fig. 5 are about two times lower than survey averages at higher masses near Fe. Figure 4 shows that below $\sim 200 \mathrm{keV}$ nucleon $^{-1}$ PSP and STEREO had Fe/O ratios roughly twice as large as ACE and Solar Orbiter, which were further away in longitude from the event site. This is consistent with the differences seen in $\mathrm{Fe} / \mathrm{O}$ ratios of large solar particle events versus events dominated by intensities near an interplanetary shock passage (Cane et al. 2006; Desai et al. 2006). The Fe/O ratios in Fig. 4 show a sharp drop above $\sim 1 \mathrm{MeV}$ nucleon ${ }^{-1}$ to values much lower than SEP survey averages at a few MeV nucleon ${ }^{-1}$ (Reames 2020). In this case, the reason is due to the lower break energy for Fe compared to $\mathrm{O}$, which leads to a sharp drop in the Fe/O ratio (Mazur et al. 1992). The nearly monotonic drop of PSP Fe/O with increasing energy covers the range of both the STEREO and ACE results; however, below $\sim 30 \mathrm{keV}$ nucleon $^{-1}$, it rises significantly above the averages. This low energy range is below most previous SEP results, and might be a new feature.

Large SEP events sometimes show enrichments of the rare isotope, ${ }^{3} \mathrm{He}$, presumably due to remnant impulsive material that is in the interplanetary suprathermal ion pool which is energized when the shock from an event like the 29 Nov. event passes through (Cohen et al. 1999; Desai et al. 2001; Mason et al. 1999). For this event, however, the high-resolution instruments on ACE and Solar Orbiter showed only upper limits at very low values $\left({ }^{3} \mathrm{He} /{ }^{4} \mathrm{He}<0.03 \%\right.$ on $\mathrm{ACE},<1 \%$ on Solar Orbiter).

The 29 Nov. 2020 large SEP event observed by the newly launched Solar Orbiter and Parker Solar Probe spacecraft 
along with previously launched missions provided multipoint observations that can be used to constrain, test, and refine global models and reveal a deeper understanding of the physical mechanisms by which large portions of the inner heliosphere are filled with radiation (Rouillard et al. 2011, 2012, 2020). As solar activity increases, and especially when Solar Orbiter and Parker Solar Probe are much closer to the Sun, additional events promise to allow critical future progress in understanding SEP acceleration and transport.

Acknowledgements. Solar Orbiter is a mission of international cooperation between ESA and NASA, operated by ESA. The Suprathermal Ion Spectrograph (SIS) is a European facility instrument funded by ESA. We thank ESA and NASA for their support of the Solar Orbiter, PSP, STEREO and ACE missions whose data were used in this Letter. Complete acknowledgements for the missions and specific instruments are too lengthy to include here but are given in the references in Table 1. Solar Orbiter and Parker Solar Probe post-launch work at JHU/APL is supported by NASA contract NNN06AA01C.

\section{References}

Band, D., Matteson, J., Ford, L., et al. 1993, ApJ, 413, 281

Cane, H. V., \& Lario, D. 2006, Space Sci. Rev., 123, 45

Cane, H. V., Reames, D. V., \& Von Rosenvinge, T. T. 1988, J. Geophys. Res., 93, 9555

Cane, H. V., Mewaldt, R. A., Cohen, C. M. S., \& Von Rosenvinge, T. T. 2006, J. Geophys. Res., 111, 11

Cohen, C. M. S., Mewaldt, R. A., Leske, R. A., et al. 1999, Geophys. Res. Lett., 26, 2697

Cohen, C. M. S., Mason, G. M., \& Mewaldt, R. A. 2017, ApJ, 843, 132
Cohen, C. M. S., Christian, E. R., Cummings, A. C., et al. 2021, A\&A, 656, A29 (SO Cruise Phase SI)

Desai, M. I., Mason, G. M., Dwyer, J. R., et al. 2001, ApJ, 553, L89

Desai, M. I., Mason, G. M., Dwyer, J., et al. 2003, ApJ, 588, 1149

Desai, M. I., Mason, G. M., Gold, R. E., et al. 2006, ApJ, 649, 470

Desai, M. I., Mason, G. M., Dayeh, M. A., et al. 2016, ApJ, 828, 106

Kollhoff, A., Kouloumvakos, A., Lario, D., et al. 2021, A\&A, 656, A20 (SO Cruise Phase SI)

Luhmann, J. G., Curtis, D. W., Schroeder, P., et al. 2008, Space Sci. Rev., 136, 117

Mason, G. M., Gold, R. E., Krimigis, S. M., et al. 1998, Space Sci. Rev., 86, 409

Mason, G. M., Mazur, J. E., \& Dwyer, J. R. 1999, ApJ, 525, L133

Mason, G. M., Korth, A., Walpole, P., et al. 2008, Space Sci. Rev., 136, 257

Mazur, J. E., Mason, G. M., Klecker, B., \& McGuire, R. E. 1992, ApJ, 401, 398

McComas, D. J., Alexander, N., Angold, N., et al. 2014, Space Sci. Rev., 204, 187

Mewaldt, R. A., Cohen, C. M. S., Cook, W., et al. 2008, Space Sci. Rev., 136, 285

Mewaldt, R. A., Looper, M. D., Cohen, C. M. S., et al. 2012, Space Sci. Rev., 171,97

Müller, D., Cyr, O. C. S., Zouganelis, I., Gilbert, H. E., \& Marsden, R. 2020, A\&A, 642, A1

Reames, D. V. 2020, Space Sci. Rev., 216, 20

Richardson, I. G., Von Rosenvinge, T. T., Cane, H. V., et al. 2014, Sol Phys., 289, 3059

Rodríguez-Pacheco, J., Wimmer-Schweingruber, R. F., Mason, G. M., et al. 2020, A\&A, 642, A7

Rouillard, A. P., Odstřcil, D., Sheeley, N. R., et al. 2011, ApJ, 735, 7

Rouillard, A. P., Sheeley, N. R., Tylka, A., et al. 2012, ApJ, 752, 44

Rouillard, A. P., Pinto, R. F., Vourlidas, A., De Groof, A., \& Thompson, W. T. 2020, A\&A, 642, A2

Stone, E. C., Frandsen, A. M., Mewaldt, R. A., et al. 1998a, Space Sci. Rev., 86,1

Stone, E. C., Cohen, C. M. S., Cook, W., et al. 1998b, Space Sci. Rev., 86, 357 


\section{Appendix A: Spectral forms and breaks}

The broken power-law spectra shapes in the Nov. 29 event are typical of large solar energetic particle events. In order to compare the event with previous surveys, we fitted the PSP, STEREO-A, and ACE spectra using the four-parameter Band function (Band et al. 1993), which has provided good fits to energetic particle spectra in the energy range studied here (Desai et al. 2016; Mewaldt et al. 2012). The Band function is in energy, $E$, and features four parameters: a normalization constant, a low-energy power law index $\left(\gamma_{\mathrm{a}}\right)$, a high-energy index, $\left(\gamma_{\mathrm{b}}\right)$, and a "break" energy $E_{\mathrm{B}}$ which is in an exponential segment $\exp \left(-E / E_{\mathrm{B}}\right)$ that connects the two power laws. The units of $E$ and $E_{\mathrm{B}}$ are in $\mathrm{MeV}$ nucleon $^{-1}$. The $\gamma_{\mathrm{a}}$ and $\gamma_{\mathrm{b}}$ give the spectral slopes far from $E_{\mathrm{B}}$ but in the case of SEP spectra in the energy range shown here, $E_{\mathrm{B}}$ is close to the range of the segments, so the power law slopes often differ from the $\gamma$ values (see discussion in Desai et al. 2016). Figure A.1 shows Band parameter fits $\gamma_{\mathrm{b}}$ vs $\gamma_{\mathrm{a}}$ from the PSP (O only), ACE (He, O, and Fe), and STEREO-A ( $\mathrm{He}, \mathrm{O}$, and $\mathrm{Fe}$ ) along with values obtained from fits for $\mathrm{H}, \mathrm{O}$, and $\mathrm{Fe}$ in the 46-event survey of Desai et al. (2016). Low energy $\left(\gamma_{\mathrm{a}}\right)$ values are well within the spread of the earlier survey. The high energy $\left(\gamma_{\mathrm{b}}\right)$ values are similar to the survey results for ACE. However, both PSP and STEREO are higher than the bulk of the survey results. This may be due to the fact that the Desai survey events were well-connected western hemisphere SEPs with no local Interplanetary Shock (IP) or Energetic Storm Particle (ESP) component, while at PSP and STEREO the intensity profiles were strongly affected by the shock (Cohen et al. 2021). In any case, the high $\gamma_{\mathrm{b}}$ values for PSP and STEREO indicate much steeper spectra than typical for the survey of Desai et al. (2016).

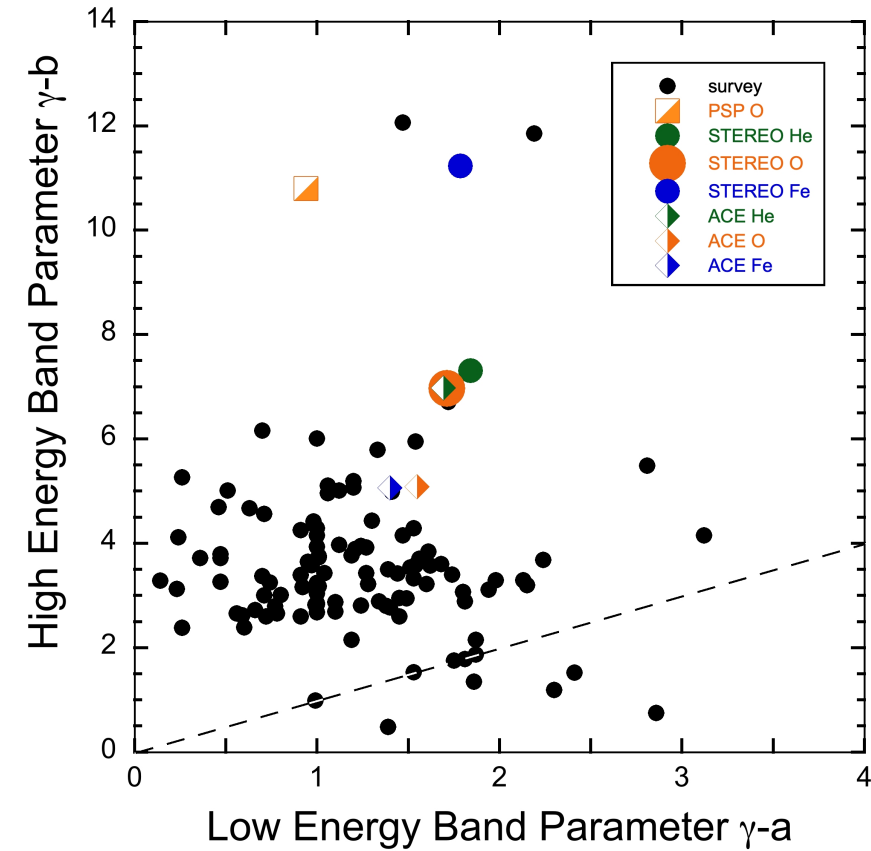

Fig. A.1. Band spectral parameters for PSP (half-filled square), STEREO-A (filled circles), and ACE (half-filled diamonds) compared to survey results for $\mathrm{H}, \mathrm{O}$ and $\mathrm{Fe}$ in 46 SEP events from Desai et al. (2016). Green points: He, orange points: O, blue points: Fe. Dashed line shows $\gamma_{\mathrm{a}}=\gamma_{\mathrm{b}}$. 